\title{
Functional Connectivity Alterations in Children with Spastic and Dyskinetic Cerebral Palsy
}

\author{
Yun Qin $\left(\mathbb{D},{ }^{1}\right.$ Yanan $\mathrm{Li}^{2}{ }^{2}$ Bo Sun, ${ }^{1}$ Hui He $\mathbb{D},{ }^{1}$ Rui Peng, ${ }^{1}$ Tao Zhang $\mathbb{D}^{1},{ }^{1}$ Jianfu Li, ${ }^{1}$ \\ Cheng Luo ${ }^{D}{ }^{1}$ Chengyan Sun ${ }^{1}{ }^{2},{ }^{2}$ and Dezhong Yao ${ }^{1}$ \\ ${ }^{1}$ The Clinical Hospital of Chengdu Brain Science Institute, MOE Key Lab for Neuroinformation, High-Field Magnetic Resonance \\ Brain Imaging Key Laboratory of Sichuan Province, University of Electronic Science and Technology of China, \\ Chengdu 610054, China \\ ${ }^{2}$ Sichuan Rehabilitation Hospital, Chengdu, China
}

Correspondence should be addressed to Cheng Luo; chengluo@uestc.edu.cn and Chengyan Sun; 279798774@qq.com

Received 16 March 2018; Revised 1 June 2018; Accepted 6 June 2018; Published 15 August 2018

Academic Editor: Carlo Cavaliere

Copyright ( 2018 Yun Qin et al. This is an open access article distributed under the Creative Commons Attribution License, which permits unrestricted use, distribution, and reproduction in any medium, provided the original work is properly cited.

Cerebral palsy (CP) has long been investigated to be associated with a range of motor and cognitive dysfunction. As the two most common CP subtypes, spastic cerebral palsy (SCP) and dyskinetic cerebral palsy (DCP) may share common and distinct elements in their pathophysiology. However, the common and distinct dysfunctional characteristics between SCP and DCP on the brain network level are less known. This study aims to detect the alteration of brain functional connectivity in children with SCP and DCP based on resting-state functional MRI (fMRI). Resting-state networks (RSNs) were established based on the independent component analysis (ICA), and the functional network connectivity (FNC) was performed on the fMRI data from 16 DCP, 18 bilateral SCP, and 18 healthy children. Compared with healthy controls, altered functional connectivity within the cerebellum network, sensorimotor network (SMN), left frontoparietal network (LFPN), and salience network (SN) were found in DCP and SCP groups. Furthermore, the disconnections of the FNC consistently focused on the visual pathway; covariance of the default mode network (DMN) with other networks was observed both in DCP and SCP groups, while the DCP group had a distinct connectivity abnormality in motor pathway and self-referential processing-related connections. Correlations between the functional disconnection and the motor-related clinical measurement in children with $\mathrm{CP}$ were also found. These findings indicate functional connectivity impairment and altered integration widely exist in children with $\mathrm{CP}$, suggesting that the abnormal functional connectivity is a pathophysiological mechanism of motor and cognitive dysfunction of CP.

\section{Introduction}

Cerebral palsy $(\mathrm{CP})$ is the most common cause of physical disability in early childhood, occurring at a rate of around 2 per 1000 live births $[1,2]$. CP encompasses a range of motor and postural disorders resulting from nonprogressive injury during the prenatal or infant development, causing serious activity limitation often accompanied by various degrees of sensation and cognition impairments $[3,4]$. Spastic cerebral palsy (SCP) is the most common CP subtype, which is usually presented with bilateral spastic or hemiplegic disability with increased muscle tone, hyperreflexia, and persistence of primitive reflexes [5]. Dyskinetic CP (DCP) is the second common subtype, affecting $15 \%-20 \%$ of children with CP
[6]. The new definition of dyskinetic CP includes dystonic and choreoathetoid $\mathrm{CP}$, which is characterized by abnormal movement or posture, with involuntary, recurring, uncontrolled, and occasionally stereotyped movements [7, 8]. Besides motor function impairment, both SCP and DCP subtypes tend to be associated with visual and auditory impairment and cognitive impairments such as intellectual and learning disability $[1,7]$. Furthermore, evidence points to abnormal sensorimotor reorganization, attention, and executive function deficits, as well as visual-perceptual impairments in children with DCP and SCP [9-11].

The wide array of dysfunction in $\mathrm{CP}$ is due to the heterogeneous nature of the underlying cerebral lesions. Depending on the location, extent, and timing of the insult, clinical 
symptoms vary largely [12]. Brain maldevelopment, white matter (WM) lesions, basal ganglia lesions, and cortical/subcortical lesions $[13,14]$ are the most common pathological findings in CP. Bilateral spastic CP (diplegia and tetraplegia) is foremost associated with white matter injury especially periventricular leukomalacia (PVL), in particular in children born preterm or prolonged hypoxic-ischemic events $[15,16]$. Some MR studies have showed that brain injury pattern of DCP was associated with basal ganglia and thalamic injury following profound hypoxic insults and commonly seen in term infants [17, 18]. Cortical injury and WM involvement have also been reported in DCP cases [13]. Nevertheless, the heterogeneity among neuroimaging studies makes it ambiguous to systematically understand the relationship between the dysfunction and the brain state in children with CP.

Previous studies have widely applied diffusion tensor imaging to focus on the microstructural damage of white matter fiber tract of CP $[19,20]$. Inverse correlation was found between the quantitative indicators of sensory-motor tracts and gross motor clinical grade in CP group [21]. In addition, some voxel-based morphometry studies have investigated that the gray matter abnormality in CP was associated with clinical features [22-24]. However, the common and distinguished brain dysfunctional characteristics associated with clinical features between DCP and SCP are relatively less explored.

Functional magnetic resonance imaging (fMRI) has been effectively used in children with $\mathrm{CP}$ to map the regional motor and cognitive processing [25-27]. Applications of fMRI in children with CP have mainly focused on the somatosensory, motor, and language tasks which are directly associated with the sensorimotor dysfunction in CP $[28,29]$. Other cognitive impairments widely existing in children with $\mathrm{CP}$, such as visual-perceptual, attention, and executive function $[9-11,30]$, are less explored because performing relative tasks during fMRI scanning may be difficult for children with CP. In contrast, the resting-state fMRI scanning is simple and easy to execute for these children [31]. Functional connectivity, referring to an analysis for identifying spatial patterns of coherent BOLD activity in distributed brain regions, has been used to detect the abnormal pathways (e.g., somatosensory, motor, and thalamocortical pathway) in children with CP using resting-state fMRI data [22, 32, 33]. For defining distinct modes of long-distance interactions, independent component analysis (ICA) has been a popular method to generate resting-state networks (RSNs) [34, 35], and resting-state functional network connectivity (FNC) [36] can be used to represent the temporal correlation among these RSNs. Therefore, investigations of the RSNs and FNC may provide more information to advance the understanding of the underlying physiopathology mechanisms in children with SCP and DCP. We hypothesized that common and distinct functional connectivity patterns may exist in SCP and DCP.

To test our hypothesis, 16 children with DCP, 18 children with SCP, and 18 healthy controls were included to explore the resting-state functional connectivity within and between RSNs. Group-level independent component analysis [37, 38] was used to extract the RSNs, and FNC analysis was performed. Group comparisons were then conducted for RSNs and FNC. The relationship between functional disconnection and motor-related clinical measurement in $\mathrm{CP}$ was also examined.

\section{Material and Methods}

2.1. Participants. A total of 43 children with CP were recruited from Sichuan Rehabilitation Hospital: 22 DCP (10 female, mean age: 9 years, age range: 3-18 years) and 21 SCP (10 female, mean age: 9.2 years, age range: $3-16$ years) were involved. The diagnosis for the two subtypes of CP was made through standardized assessment by neurologists based on clinical features and MRI scanning [7]. All children with SCP were diplegic, and all children with DCP were dystonic. The inclusion criteria for the study were as follows: (1) a diagnosis of DCP and SCP with predominant spastic diplegic or dyskinetic features, (2) age under 18 years old, and (3) no history of trauma or brain operation. Children with serious brain loss or lesions (lesion size $>1 \mathrm{~cm}^{3}$, including 4 DCP children and 2 SCP children) were excluded based on MRI brain image. No child was on medication. 20 healthy children without history of neurological disorder or brain injury (7 female, mean age: 9.3 , age range: 5-12) were included in the healthy control group (HC). Parents of all participants gave written informed consent in accordance with the Declaration of Helsinki. This study was performed according to the guidelines approved by the Ethics Committee of the University of Electronic Science and Technology of China (UESTC).

2.2. Clinical Measurement. Motor function was assessed for the DCP and SCP groups by the Gross Motor Function Classification System (GMFCS) [39]. GMFCS scores range from level I, which indicates children with no disability for community mobility, to level $\mathrm{V}$, which includes children who are totally dependent on assistance for mobility. Everyday activities of daily living (ADL) [40] were also evaluated by the Assessment of Motor and Process Skills (AMPS) to access the self-care ability during everyday life. Both GMFCS and ADL evaluations were conducted by physical therapists in the Rehabilitation Assessment Department, Sichuan Rehabilitation Hospital.

2.3. Imaging Data Acquisition. Images were acquired on a $3 \mathrm{~T}$ MRI scanner (GE Discovery MR750) at the MRI Research Center of UESTC. Children with CP underwent MRI scanning under monitored sedation induced by midazolam $(0.1 \mathrm{mg} / \mathrm{kg})$ and propofol $(1-2 \mathrm{mg} / \mathrm{kg})$, which is approved by the Department of Anaesthesia of the Sichuan Rehabilitation Hospital, and written informed consent was obtained from participants' parents. The HC children were instructed simply to keep their eyes closed and remain still without sedation. The same scanning protocol was used for both CP and HC children as follows. Highresolution T1-weighted images were acquired using a 3dimensional fast spoiled gradient-echo (T1-3D FSPGR) sequence (repetition time $(\mathrm{TR})=5.956 \mathrm{~ms}$, echo time $(\mathrm{TE})=1.964 \mathrm{~ms}$, flip angle $(\mathrm{FA})=9^{\circ}, \quad$ matrix $=256 \times 256$, 
field of view $(F O V)=25.6 \times 25.6 \mathrm{~cm}^{2}$, slice thickness $=1 \mathrm{~mm}$, no gap, 152 slices). T2 images were also acquired using $\mathrm{OAx}$ T2 fluid-attenuated inversion recovery (OAx T2 FLAIR) $\left(\mathrm{TR}=8400 \mathrm{~ms}, \mathrm{TE}=150 \mathrm{~ms}, \mathrm{FA}=111^{\circ}\right.$, matrix $=256 \times 256$, FOV $=25.6 \times 25.6 \mathrm{~cm}^{2}$, slice thickness $=4 \mathrm{~mm}$ ). Resting-state functional MRI data were acquired using gradient-echo echo-planar imaging sequences $(\mathrm{TR}=2000 \mathrm{~ms}, \mathrm{TE}=30 \mathrm{~ms}$, $\mathrm{FA}=90^{\circ}$, matrix $=64 \times 64, \mathrm{FOV}=24 \times 24 \mathrm{~cm}^{2}$, slice thickness $/$ gap $=4 \mathrm{~mm} / 0.4 \mathrm{~mm}$ ), with an eight channel-phased array head coil. A total of 255 volumes were collected from each participant.

2.4. fMRI Preprocessing. Data preprocessing was performed using SPM8 (statistical parametric mapping, http://www.fil .ion.ucl.ac.uk/spm/). The first ten volumes were discarded for the magnetization equilibrium from all fMRI scans. Then, the remaining images were slice-timing corrected and realigned (motion-corrected). The transition and rotation parameters were checked, and given the relatively large head motion of the children or teenagers during fMRI scanning, we increased the motion threshold to $3 \mathrm{~mm}$ for head movement and $3^{\circ}$ for head rotation as the exclusion standard just as other studies did with adolescent participants [41-43]. Group comparison was performed for the individual mean framewise displacement (FD) calculated by averaging the relative displacement from every time point for each subject [44], and no difference was found between the groups (oneway ANOVA, $P=0.2439$ ). The formula to calculate the FD is

$\mathrm{FD}=\frac{1}{M-1} \sum_{i=2}^{M} \sqrt{\left|\Delta t_{x_{i}}\right|^{2}+\left|\Delta t_{y_{i}}\right|^{2}+\left|\Delta t_{z_{i}}\right|^{2}+\left|\Delta d_{x_{i}}\right|^{2}+\left|\Delta d_{y_{i}}\right|^{2}+\left|\Delta d_{z_{i}}\right|^{2}}$,

where $M$ is the length of the time courses; $x_{i}, y_{i}$, and $z_{i}$ are translations/rotations at the ith time point in the $x, y$, and $z$ directions, respectively; $\Delta t$ represents the framewise displacement translation; $\Delta d$ represents the framewise displacement rotation; and $\Delta d_{x_{i}}=x_{i}-x_{i-1}$, similar for $\Delta d_{y i}, \Delta d_{z i}, \Delta t_{x i}, \Delta$ $t_{y i}$, and $\Delta t_{z i}$.

Spatial normalization was performed using the T1-based transformation. The individual $\mathrm{T} 1$ images were coregistered to the functional images and then segmented and normalized to the Montreal Neurologic Institute (MNI) space by a 12 parameter nonlinear transformation. Additionally, we used a cost-function modification to exclude the lesion area avoiding bias during spatial normalization [45]. The process has been implemented in SPM8 and adopted in other brain imaging studies with lesions in our lab [46]. The transformation parameters were applied to functional images. Then, functional data were resampled to $3 \times 3 \times 3 \mathrm{~mm}^{3}$ voxels after spatial normalization. Moreover, the images were spatially smoothed through convolution with a $6 \mathrm{~mm}$ full-width half-maximum (FWHM) Gaussian kernel.

2.5. Lesion Mapping. In this study, we constructed a lesion overlap image of CP. First, a radiologist marked the gray matter lesions on individual 3D T1 images. The lesions in this study mainly covered some voxels in the occipital region for one child and basal ganglia region for four children. Then, the union of all individual lesions was used to construct a group lesion mask after the spatial normalization process. At last, a specific group mask was generated from the gray matter template excluding the patients' group lesion mask for the next ICA analysis.

2.6. Independent Component Analysis. As a data-driven statistical analysis technique, ICA processing yields independent components (ICs) which represent a group of brain regions with a unique pattern of synchronized neural activity. Components with special spatial pattern can be selected as resting-state networks. For all $\mathrm{CP}$ children and healthy controls, group ICA analysis was performed to decompose the data into ICs using GIFT software [38] (version 2.0e; http:// mialab.mrn.org/software/gift/). The specific group mask excluding the CP lesion mask was used in the group ICA. Principal component analysis (PCA) was adopted for the reduction of data dimensionality. The number of ICs was determined according to the minimum description length (MDL) [47]. In ICASSO (http://research.ics.tkk.fi/ica/ icasso), the infomax algorithm was repeated 20 times as an independent component estimation. Then, the dualregression (DR) approach was used in the back reconstruction step to back reconstruct the individual participant components [48]. Thus, IC time courses and spatial maps were acquired for each participant, and the participant-specific maps were converted to $Z$-score. In addition, for the validation of the selection of the separated ICs, we implemented the ICA using different independent component numbers (model order) and severally conducted the following analysis (i.e., within RSN analysis and FNC analysis) for seven times.

2.7. Within RSN Analysis. Among the 39 components resulting from ICA, 14 components were selected as nonartifactual RSNs through visual inspection in accordance with previously published results [49-52]. For each of the RSNs, Z-maps in each group were firstly gathered using the one-sample $t$-test for revealing group main effects, and the resulting statistical map was thresholded at $P<0.05$ using the false discovery rate (FDR) correction. Then, group comparison was conducted for the $Z$-maps of the RSN using one-way ANOVA restricted to the voxels within a union mask, which was defined by the one-sample $t$-test results in three groups. Between-group effects were thresholded at $P<0.05$ with voxel-wise FDR correction, and the minimum cluster size was 25 voxels. Regions $\left(3^{*} 3^{*} 3\right.$ voxels) with high significant difference in ANOVA were chosen from each RSN and used in the post hoc analysis. During the one-way ANOVA and post hoc analysis, age and sex, as well as headmotion variables, were treated as unconcerned covariates.

2.8. FNC Analysis between RSNs. After ICA, the individuallevel time courses of the identified RSNs were derived from the spatial-temporal dual regression. In order to investigate the relationship between time courses of different RSNs, FNC analysis was performed. First, temporal band-pass filter (band pass $0.01-0.1 \mathrm{~Hz}$ ) was used to reduce the effects of lowfrequency drift and high-frequency physiological noise on the time courses. Then, correlations were computed between 
the time courses of any two of the RSNs for each participant. Thus, individual potential internetwork connections (91 connections) were generated. After Fisher $Z$-translation of the correlation coefficients, one-sample $t$-test $(P=0.05$, corrected by FDR) was applied to examine the significant temporal interactions between any two RSNs in DCP, SCP, and $\mathrm{HC}$ groups, respectively. To better understand the group difference of FNC, one-way ANOVA was performed for all potential connections between RSNs, with the significance cutoff $P<0.05$ corrected by FDR to control for multiple comparisons. Then, the post hoc comparison $(P<0.01)$ was used to the connections with statistical significance in the ANOVA between groups. During the one-way ANOVA and post hoc analysis, age and sex, as well as head-motion variables, were treated as unconcerned covariates.

2.9. Correlations with Clinical Measurement. Correlations were calculated between the functional connectivity in RSNs/FNC and the clinical measurements of CP. For each RSN, the regions $(3 * 3 * 3$ voxels) with a peak $F$ value in the ANOVA were selected as regions of interest (ROIs), and the coordinates of the ROIs were extracted. Then, the mean $Z$-scores within the ROI was used for the following correlation calculation. In addition, the coefficients of FNC with high significance in ANOVA were also used to calculate the correlation with the GMFCS and ADL scores.

2.10. Statistical Analyses. Before statistical analyses, normality of the data distribution was tested using the Lilliefors test, including the age, ADL scores, $Z$-map of RSNs for each voxel, and FNC connections. Then, to investigate the group difference for demographic and clinical data, chi-square test was applied to the categorical data including gender and GMFCS scores; one-way ANOVA and two-sample $t$-test were used for age and ADL scores, respectively. For RSN and FNC analysis, group comparison among the three groups was performed using one-way ANOVA. Significant differences revealed by ANOVA $(P<0.05$, corrected by FDR) were further analyzed for multiple comparisons using Tukey's post hoc test. In addition, relationship between functional connectivity and clinical scores was examined using Pearson correlation for ADL and Spearman correlation for GMFCS, with statistical significance level $P<0.05$ corrected by FDR. The voxel-level statistical analysis of RSNs was conducted using SPM8 (statistical parametric mapping), and the other statistical analysis, including the FNC group comparison and the correlation, was conducted using MATLAB functions (MATLAB 2015).

\section{Results}

3.1. Participants and Clinical and Radiological Findings. After the fMRI data head-motion checking, the final cohort in this study consisted of 16 DCP, 18 SCP, and 18 HC children. No significant difference was found for age and gender among the three groups (one-way ANOVA for age, $P=0.818$; chisquare test for gender, $P=0.907)$. Comparing to SCP, DCP showed higher GMFCS (chi-square test, $P=0.003$ ) and lower ADL (two-sample $t$-test, $P<0.001$ ), indicating more severe motor and daily activity disability in the DCP group. Cerebral abnormalities were evaluated by two radiologists according to the high-resolution T1 (3D FSPGR) and T2 (OAx T2 FLAIR) images. Among 36 children, 5 children have gray matter lesions involving 1 child with small occipital lesion and 4 children with basal ganglia abnormality, mainly located in the bilateral putamen. 21 children have predominant white mater lesion types consisting of periventricular leukomalacia, ventricular enlargement, and other local white matter lesions. Demographic and clinical data of the sample were shown in Table 1.

3.2. RSN Identifications. Fourteen components were selected as the resting-state relevant networks from the group ICA in accordance with the previously published results $[35,51]$. No clusters in each RSN fell within the lesion of any of the children. The spatial maps of the 14 RSNs are illustrated in Figure 1. These networks are labeled as follows: cerebellum: the spatial patterns primarily encompassed the cerebellum posterior lobe and declive; SMN1: sensorimotor network included the paracentral lobule, the supplementary motor area, and the pre- and postcentral gyrus; SMN2: sensorimotor network focused at the bilateral primary somatosensory cortex, including pre- and postcentral gyrus areas; DAN: dorsal attention network mainly included the bilateral intraparietal sulcus, frontal eye field, and middle temporal lobe; antDMN: the anterior part of default mode network $(\mathrm{DMN})($ antDMN) included the superior frontal gyrus and middle frontal gyrus; postDMN: the posterior part of DMN involved the posterior cingulate cortex (PCC), precuneus, and bilateral angular gyrus; $S R N$ : the self-referential network mainly included the anterior cingulate and bilateral medialventral prefrontal cortex; primVN: the primary visual network showed the spatial patterns consisting of the cuneus, calcarine, and lateral lingual gyrus; extraVN: the extrastriate visual network encompassed the bilateral fusiform gyrus, middle temporal, and middle occipital areas; $A N$ : the auditory network primarily encompassed middle temporal gyrus and superior temporal gyrus corresponding to the auditory system; LFPN: the left lateral frontoparietal network involved the left middle frontal gyrus, inferior parietal lobule, superior parietal lobule, and angular gyrus; $R F P N$ : the right lateral frontoparietal network showed the similar spatial patterns with LFPN. LFPN and RFPN were the only maps strongly lateralized and left-right mirrors of each other; $S N$ : the salience network showed spatial patterns mainly consisting of dorsal anterior cingulate (dACC) and orbital frontoinsular cortices, as well as part of prefrontal areas; $C E N$ : the central executive network showed spatial patterns comprising the superior and middle frontal cortices, anterior cingulate, and paracingulate gyri. The group-level spatial maps of 14 RSNs for HC, SCP, and DCP were shown in Supplementary Material Figure S1.

3.3. Group Comparisons of Functional Connectivity within RSNs. Group comparison of functional connectivity within RSNs was performed using one-way ANOVA. Significant difference among the DCP, SCP, and HC groups was found within four RSNs $(P<0.05$, corrected by FDR), 
TABLE 1: Demographic and clinical data of the sample.

\begin{tabular}{|c|c|c|c|c|}
\hline & DCP & SCP & $\mathrm{HC}$ & $P$ value \\
\hline Number of participants (N) & 16 & 18 & 18 & \\
\hline Age (mean years \pm std) & $9.6 \pm 5.0$ & $8.9 \pm 3.1$ & $9.5 \pm 2.2$ & 0.818 \\
\hline Gender & $10 \mathrm{M}, 6 \mathrm{~F}$ & $10 \mathrm{M}, 8 \mathrm{~F}$ & $11 \mathrm{M}, 7 \mathrm{~F}$ & 0.907 \\
\hline GMFCS & II: 3, III: 7, IV: 5, V: 1 & I: 9, II: 5, III: 4 & & 0.003 \\
\hline ADL (mean scores \pm std) & $38.44 \pm 18$ & $82.55 \pm 3.4$ & & $<0.001$ \\
\hline \multicolumn{5}{|l|}{ Neuroimage finding $(N)$} \\
\hline White matter lesion & 6 & 15 & & \\
\hline Cortical gray matter abnormality & 1 & 0 & & \\
\hline Basal ganglia/thalamus abnormality & 3 & 1 & & \\
\hline Normal $(N)$ & 6 & 2 & & \\
\hline
\end{tabular}

M: male; F: female; std: standard deviation; $N$ : number of participants. $P$ value: comparisons for age and gender among the three groups: the variable gender was analyzed using chi-square test, while the age was analyzed using ANOVA; comparisons for GMFCS and ADL between DCP and SCP: the variable GMFCS was analyzed using chi-square test, while the ADL were analyzed using two-sample $t$-test.
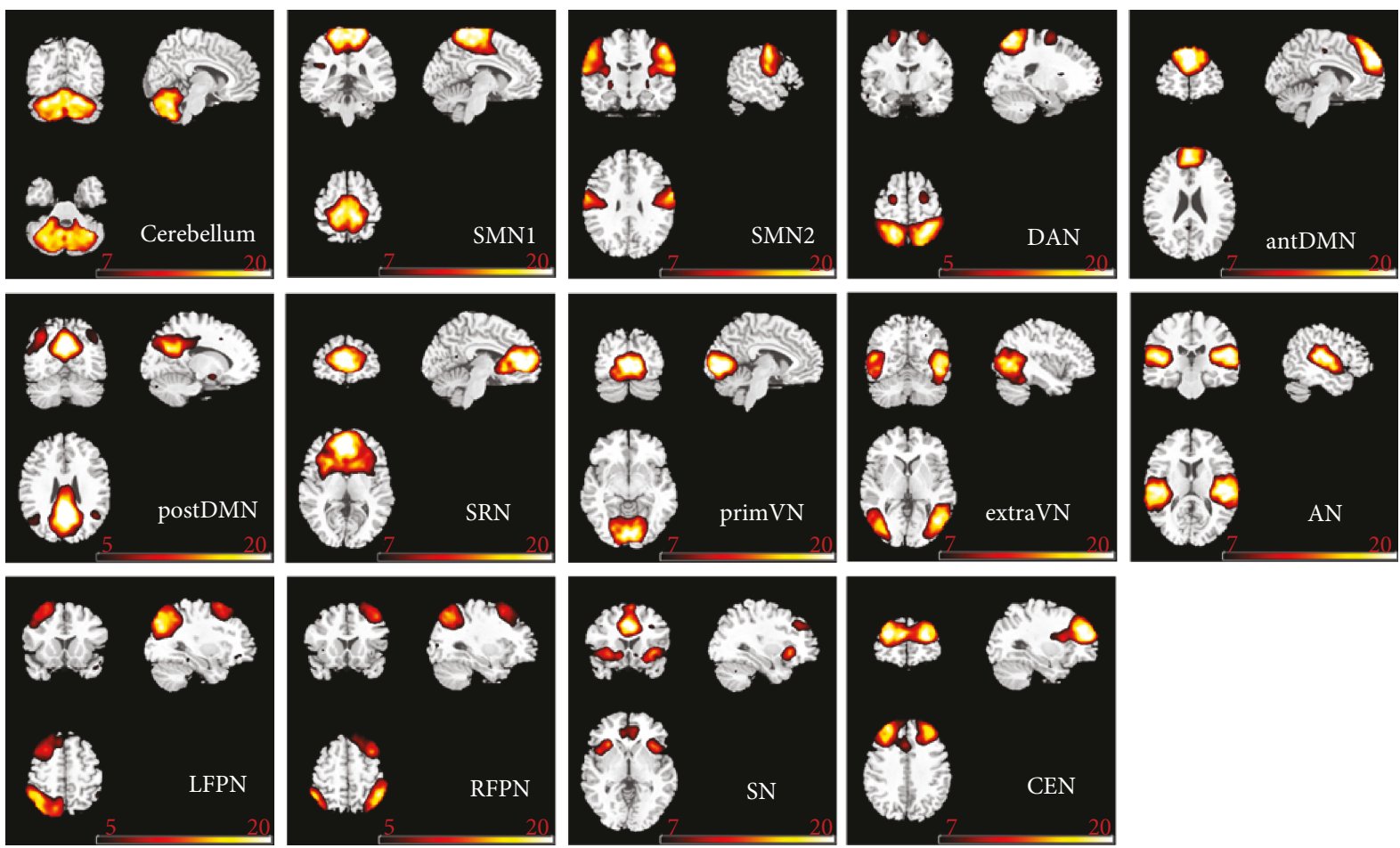

FIgURE 1: The spatial patterns of 14 RSNs identified according to the group ICA in all children. SMN: sensorimotor network; DAN: dorsal attention network; antDMN: the anterior part of default mode network; postDMN: the posterior part of default mode network; SRN: the self-referential network; primVN: the primary visual network; extraVN: the extrastriate visual network; AN: the auditory network; LFPN: the left lateral frontoparietal network; RFPN: the right lateral frontoparietal network; SN: the salience network; CEN: the central executive network.

including the cerebellum network, SMN2, LFPN, and SN (Table 2). Post hoc analysis between the groups was performed within the regions where significant difference was observed in the three groups (Table 3, Table 4). Figure 2 showed differences between the groups. Compared with HC, both DCP and SCP illustrated decreased functional connectivity within the cerebellum network, SMN2, and LFPN. Moreover, the SN revealed not only the reduced functional connectivity in middle frontal and superior frontal gyrus but also the increased functional connectivity in the anterior cingulum (ACC) in CP. No significant difference was found for functional connectivity within RSNs between the DCP and SCP group. In addition, according to the validation analysis using different independent component numbers in ICA processing, the results of functional connectivity within RSNs were mostly repeated in calculations seven times (details shown in Supplementary Material Figure S2). 
TABLE 2: Significant differences of functional connectivity within four RSNs in one-way ANOVA comparison among the three groups (ANOVA, $P<0.05$ FDR-corrected).

\begin{tabular}{|c|c|c|c|c|c|c|}
\hline \multirow{2}{*}{ Networks } & \multirow{2}{*}{ AAL regions } & \multicolumn{3}{|c|}{ MNI coordinates } & \multirow{2}{*}{ Peak $F$ value } & \multirow{2}{*}{ Cluster voxels } \\
\hline & & $x$ & $y$ & $z$ & & \\
\hline \multirow{5}{*}{ Cerebellum } & Cerebelum_Crus1_R & 27 & -78 & -36 & 31.3056 & 63 \\
\hline & Cerebelum_Crus2_R & 33 & -70 & -38 & 19.3594 & 76 \\
\hline & Cerebelum_8_R & 28 & -65 & -45 & 20.8600 & 54 \\
\hline & Cerebelum_Crus2_L & -15 & -78 & -33 & 19.4947 & 49 \\
\hline & Cerebelum_Crus1_L & -20 & -74 & -34 & 17.5934 & 43 \\
\hline \multirow[t]{2}{*}{ SMN2 } & Postcentral_L & -54 & -6 & 27 & 24.0388 & 25 \\
\hline & Parietal_Inf_L & -36 & -72 & 39 & 21.8592 & 80 \\
\hline \multirow[t]{2}{*}{ LFPN } & Angular_L & -38 & -66 & 43 & 16.813 & 37 \\
\hline & Occipital_Mid_L & -38 & -69 & 41 & 19.2070 & 20 \\
\hline \multirow{4}{*}{$\mathrm{SN}$} & Cingulum_Ant_L & -6 & 30 & 15 & 20.2391 & 47 \\
\hline & Frontal_Mid_R & 33 & 36 & 30 & 17.3654 & 58 \\
\hline & Frontal_Sup_L & -18 & 39 & 36 & 22.8588 & 43 \\
\hline & Frontal_Mid_L & -30 & 34 & 37 & 14.0513 & 39 \\
\hline
\end{tabular}

MNI: Montreal Neurologic Institute; AAL: anatomical automatic labeling.

TABLE 3: Significant differences of functional connectivity within four RSNs between DCP and HC $(P<0.001)$.

\begin{tabular}{|c|c|c|c|c|c|c|}
\hline \multirow{2}{*}{ Networks } & \multirow{2}{*}{ AAL regions } & \multicolumn{3}{|c|}{ MNI coordinates } & \multirow{2}{*}{ Peak $T$ value } & \multirow{2}{*}{ Cluster voxel } \\
\hline & & $x$ & $y$ & $z$ & & \\
\hline \multirow{5}{*}{ Cerebellum } & Cerebelum_Crus1_R & 27 & -78 & -36 & -6.9746 & 65 \\
\hline & Cerebelum_Crus2_R & 22 & -74 & -39 & -5.2359 & 74 \\
\hline & Cerebelum_8_R & 21 & -69 & -40 & -5.5828 & 47 \\
\hline & Cerebelum_Crus2_L & -15 & -78 & -33 & -5.8267 & 47 \\
\hline & Cerebelum_Crus1_L & -20 & -73 & -34 & -5.1369 & 54 \\
\hline SMN2 & Postcentral_L & -51 & -9 & 27 & -6.5683 & 25 \\
\hline \multirow{2}{*}{ LFPN } & Parietal_Inf_L & -36 & -71 & 42 & -5.0843 & 54 \\
\hline & Angular_L & -39 & -66 & 42 & -5.6904 & 36 \\
\hline \multirow{3}{*}{ SN } & Frontal_Mid_R & 30 & 36 & 27 & -6.3314 & 48 \\
\hline & Frontal_Sup_L & -18 & 39 & 36 & -7.1288 & 38 \\
\hline & Frontal_Mid_L & -30 & 34 & 38 & -4.3143 & 39 \\
\hline
\end{tabular}

MNI: Montreal Neurologic Institute; AAL: anatomical automatic labeling.

TABLE 4: Significant differences of functional connectivity within four RSNs between SCP and HC $(P<0.001)$.

\begin{tabular}{|c|c|c|c|c|c|c|}
\hline \multirow{2}{*}{ Networks } & \multirow{2}{*}{ AAL regions } & \multicolumn{3}{|c|}{ MNI coordinates } & \multirow{2}{*}{ Peak $T$ value } & \multirow{2}{*}{ Cluster voxels } \\
\hline & & $x$ & $y$ & $z$ & & \\
\hline \multirow{3}{*}{ Cerebellum } & Cerebelum_Crus1_R & 24 & -75 & -33 & -5.5275 & 26 \\
\hline & Cerebelum_Crus2_R & 29 & -66 & -41 & -5.2380 & 27 \\
\hline & Cerebelum_8_R & 28 & -66 & -44 & -5.4002 & 42 \\
\hline SMN2 & Postcentral_L & -51 & -6 & 30 & -5.722 & 25 \\
\hline LFPN & Parietal_Inf_L & -45 & -54 & 51 & -6.7446 & 78 \\
\hline \multirow{3}{*}{ SN } & Cingulum_Ant_L & -3 & 33 & 15 & 6.9291 & 47 \\
\hline & Frontal_Mid_R & 33 & 36 & 36 & -5.3502 & 49 \\
\hline & Frontal_Sup_L & -24 & 39 & 36 & -5.9748 & 30 \\
\hline
\end{tabular}

MNI: Montreal Neurologic Institute; AAL: anatomical automatic labeling. 
(A) Result of ANOVA in the SMN2

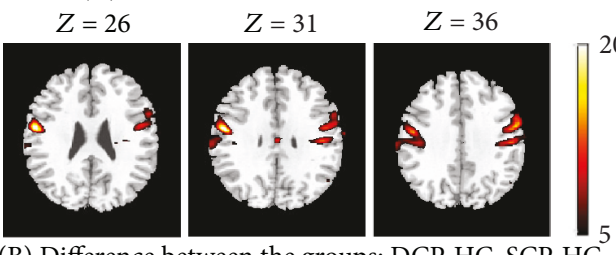

(B) Difference between the groups: DCP-HC, SCP-HC

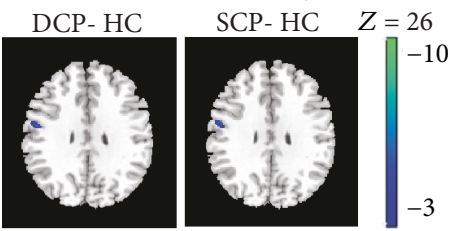

(C) Difference in regions of the SMN2 between the groups

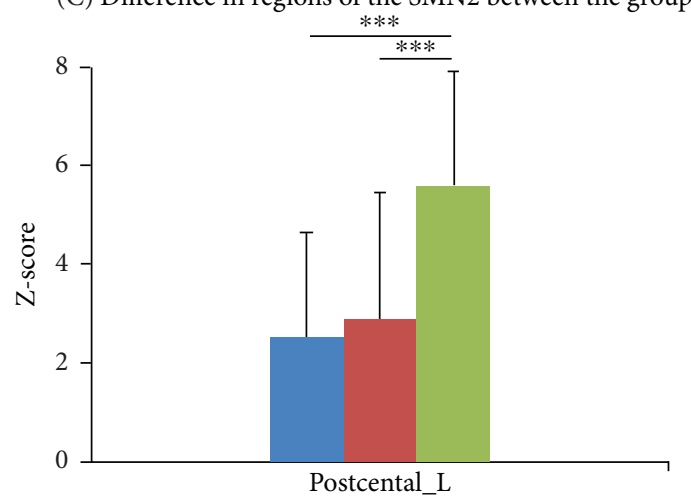

- DCP

- SCP

- $\mathrm{HC}$

(a)

(C) Difference in regions of the cerebellum between the groups $Z=28$ Result of ANOVA in the cerebellum

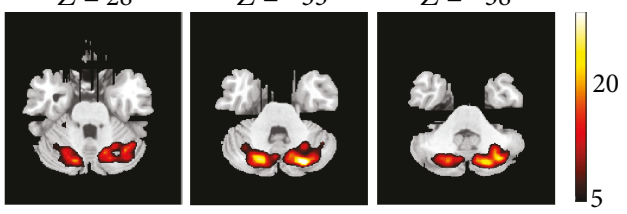

(B) Difference between the groups: DCP- HC, SCP-HC DCP- HC

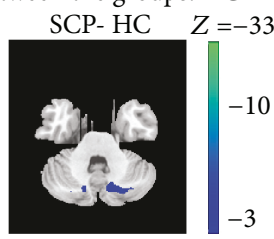

(A) Results of ANOVA in the LFPN
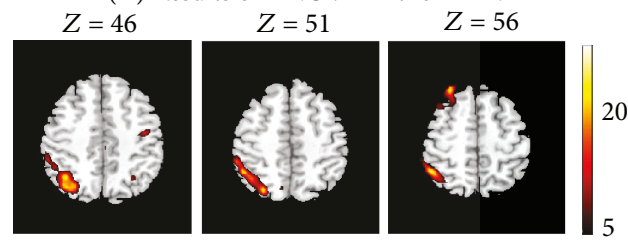

(B) Difference between the groups: DCP- HC, SCP-HC DCP-HC

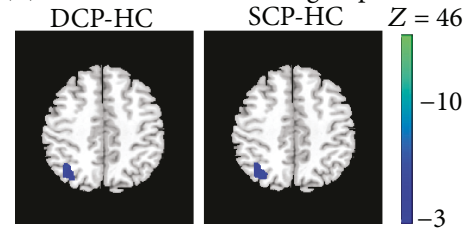

(b)

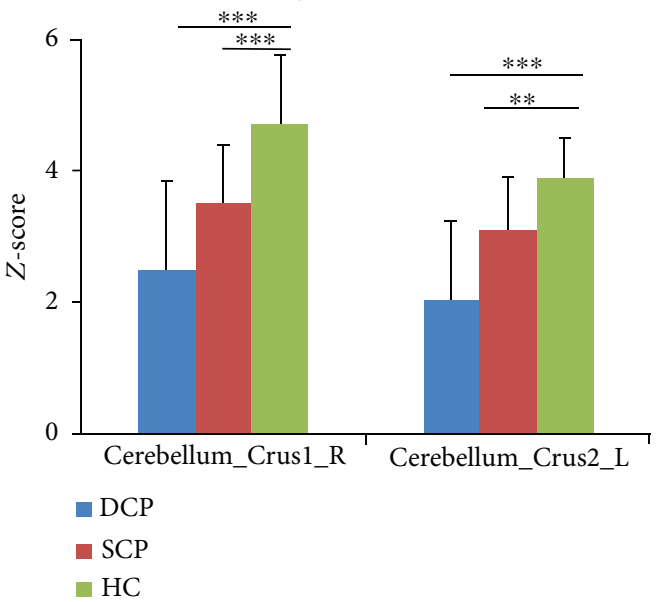

(C) Difference in regions of the LFPN between the groups

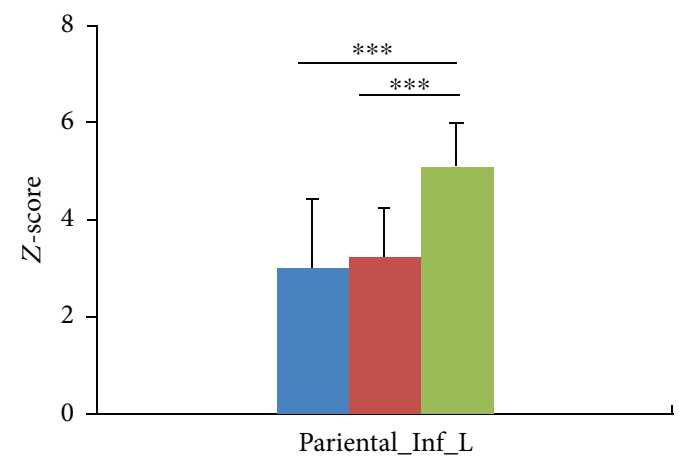

$$
\begin{aligned}
& \text { DCP } \\
& \text { SCP } \\
& \text { - } \mathrm{HC}
\end{aligned}
$$

(c)

Figure 2: Continued. 
(A) Results of ANOVA in the SN

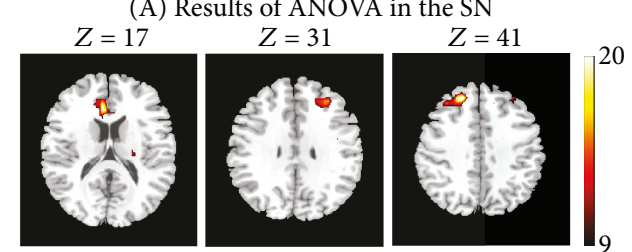

(B) Difference between the groups: DCP- HC, SCP-HC

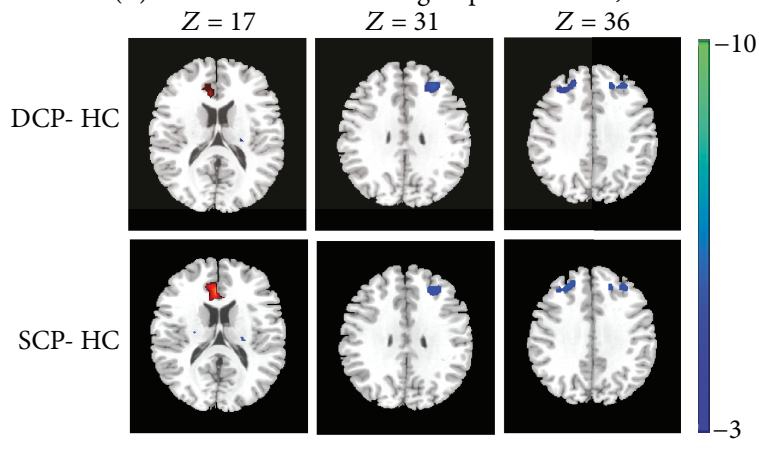

(C) Difference in regions of SN between the groups

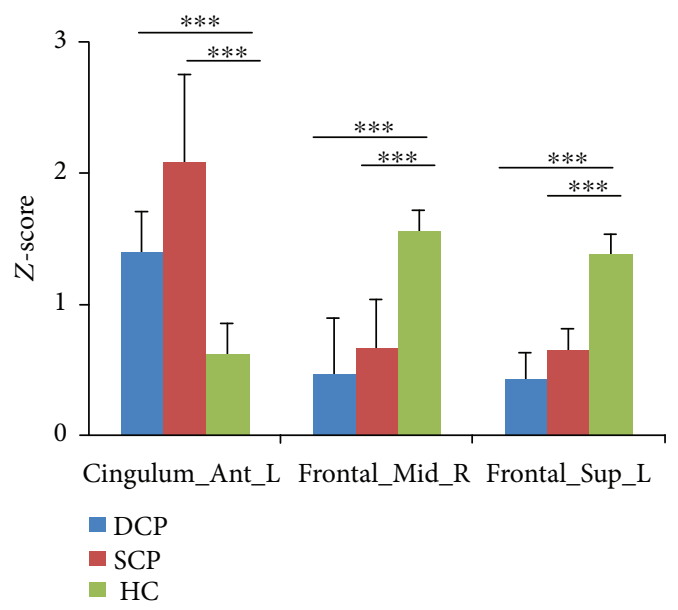

(d)

Figure 2: (a-d) Group comparison within four RSNs (SMN2, cerebellum, LFPN, and SN). (a) Significant difference was found in RSNs among the three groups. This result was achieved by performing one-way ANOVA on the maps of the three groups, with a threshold of corrected $P<0.05$. (b) Differences were obtained between the DCP and HC group, as well as between the SCP and HC group, by performing post hoc test on the RSN maps $(P<0.001)$. (c) The bar maps present the between-group differences in the regions showing significant group difference. In the bar maps, ${ }^{* *} P<0.01,{ }^{* * *} P<0.001$.

3.4. FNC Analysis between Groups. For FNC analysis, significant internetwork connections were found for each group based on the one-sample $t$-test $(P<0.05$, FDR-corrected) (Figure 3(a)). A large number of positive associations were detected between RSNs, and a small number of negative connectivity existed. Then, one-way ANOVA showed significant difference among the groups, and six connections were found to be significantly altered $(P<0.05$, FDRcorrected), including the primVN-extraVN connection, primVN-RFPN connection, RFPN-cerebellum connection, antDMN-SRN connection, postDMN-LFPN connection, and postDMN-SN connection. Moreover, in the DCP group, all of the six connections were impaired (correlation coefficient approaching zero) compared with $\mathrm{HC}$, while in the SCP group, only four out of the six disconnections existed. Comparing to SCP, DCP gave rise to more serious deficiency in the RFPN-cerebellum connection and antDMN-SRN connection. The altered FNCs were mostly involved in the validation analysis using different IC numbers (details shown in Supplementary Material Figure S3).

3.5. Relationship between Functional Connectivity and Clinical Scores. Comparisons for clinical scores between the DCP and SCP group showed that GMFCS scores in the DCP group were significantly higher than those in the SCP group, and ADL scores in the DCP group were significantly lower than those in the SCP group. Correlations were performed between the mean $Z$-scores of seven ROIs in the four RSNs (Figure 2) and clinical scores, and significant negative correlations were found between the cerebellum crus and GMFCS (Figure 4(a)). Then, correlations were performed between the FNC coefficients ( 6 connections) and the clinical measurement in the CP group. Three out of the six connections, including primVN-extraVN and postDMN-SN, as well as antDMN-SRN, were found having negative correlations with GMFCS. Moreover, three connections including primVN-RFPN, RFPN-cerebellum, and antDMN-SRN were found having significant positive correlations with ADL scores (Figures 4(b)-4(c)). Besides the correlations in the whole group, relationship between functional connectivity and clinical scores was also investigated in the subgroups (Supplementary Material Figure S4).

\section{Discussion}

In this study, we investigated the functional connectivity intra- and inter-RSNs in CP. Compared with HC, altered functional connectivity was found within the cerebellum, SMN2, LFPN, and SN networks for both the SCP and DCP groups. For FNC analysis, four functional disconnection inter-RSNs were observed in SCP, while six functional disconnection inter-RSNs were observed in DCP. The DCP and SCP groups showed different levels of aberrant connectivity. Furthermore, correlations between the functional disconnection and GMFCS/ADL scores were found. These findings indicate functional connectivity impairment, and altered integration widely exists in children with $\mathrm{CP}$, and exploring the common and distinct functional connectivity patterns may contribute to our understanding of the neuropathophysiological mechanism of different CP subtypes.

Functional connectivity analysis alteration within RSNs may elucidate the abnormal intrinsic interaction in a certain spatial pattern $[34,53]$. In this study, decreased functional connectivity was showed within two motor-related networks, 

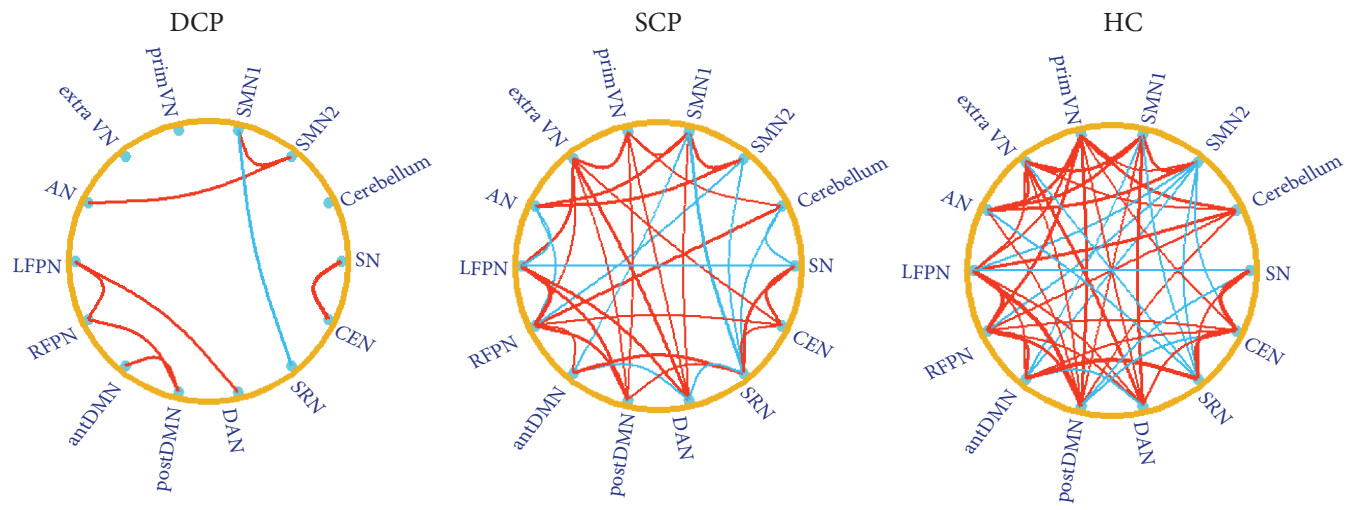

(a) FNC in the three groups
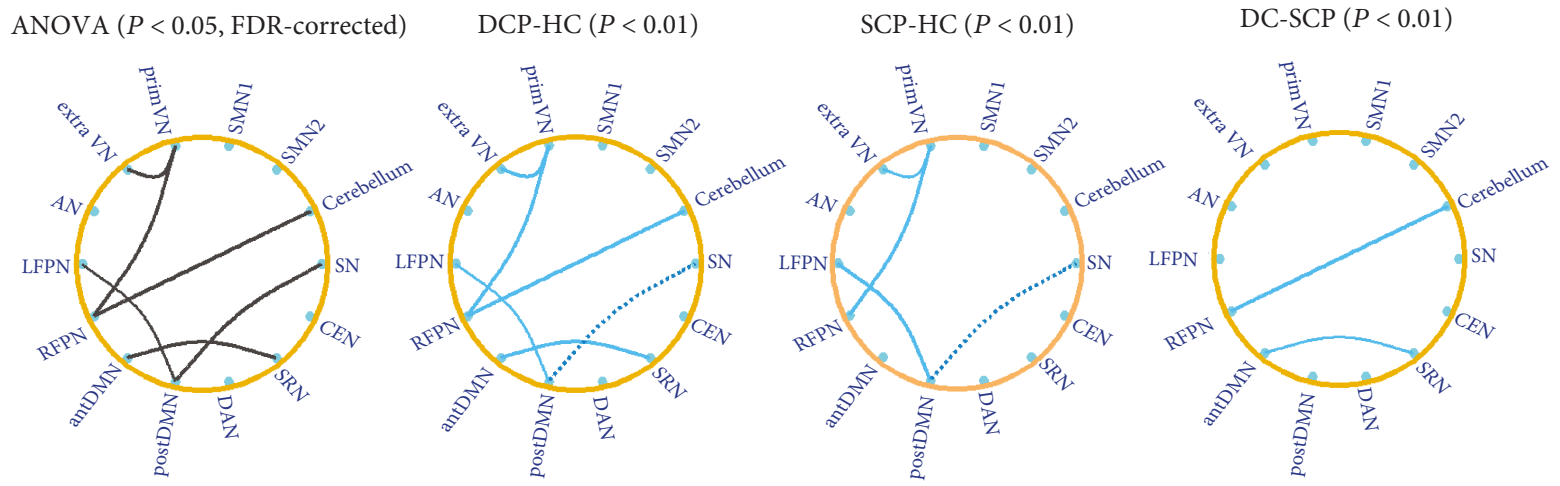

(b) Comparison results of FNC among the groups

FIgURE 3: (a) The functional network connectivity in the DCP, SCP, and HC group. The red line represents the connection with positive correlation, and the blue line represents the connection with negative correlation. The results were obtained by the one-sample $t$-test with a threshold of corrected $P<0.05$. (b) The comparison results of the functional network connectivity between the groups. The black line in the ANOVA result represents the altered connection among the three groups. Differences of functional network connectivity between DCP and HC and between SCP and HC, as well as between DCP and SCP, were observed. The blue solid line represents the connection with decreased positive FNC in CP children; and the blue dotted line represents the connection with decreased negative FNC in CP children.

that is, the cerebellum and SMN2 in children with CP. The role of the cerebellum has been well known to be involved in both motor learning and cognitive processing, and cerebellar injury might bring about posture and movement impairment [54-56]. Previous studies have demonstrated that children with CP had smaller volumes of the cerebellar hemispheres compared to controls [57]. Chronic cerebellar stimulation applied to the superomedial cortex has been used to reduce generalized cerebral spasticity, athetoid movements, and seizures [58]. Negative correlation between the cerebellum network and GMFCS in the current study suggested that decreased functional connectivity in the cerebellum would aggravate motor dysfunction in children with CP. As part of sensorimotor-related network, SMN2 focused on the primary motor and somatosensory areas, which were vulnerable regions in CP. Disrupted sensorimotor integration has been considered as a key factor that underlies motor function in CP and other movement disorders [22, 59, 60]. In this study, the decreased connectivity near the central sulcus indicated that defective sensorimotor organization could be a relevant pathophysiological element resulting to motor dysfunction in CP.
The LFPN and SN are two functional networks referred to the task activation ensemble. Frontoparietal network is an important network in spatial attention [61], and LFPN has been mentioned to correspond well to cognitionlanguage paradigms [31]. Studies about mortality and adverse neurological outcome in preterm infants with periventricular hemorrhagic infarction (PVHI) found that extended frontoparietal lesions were associated with the development of cerebral palsy $[62,63]$. Therefore, the dysfunction within the frontoparietal network might contribute to the cognitive impairment in $\mathrm{CP}$. The salience network $(\mathrm{SN})$ is a large-scale paralimbic network with coactivation in response to signal for behavioral change need [64, 65]. As part of the $\mathrm{SN}$, prefrontal cortex (PFC) plays an important role in goal-relevant top-down control, and a previous study has reviewed that PFC was engaged in the executive control adaption [66, 67]. In addition, the ACC has been proposed to serve in monitoring and detecting conflict, as well as error compensation, which was one useful drivers to adjust the level of executive control $[66,68]$. As evidences pointed to executive function impairment in children with CP [30], the reduced connectivity within the prefrontal cortex in this 

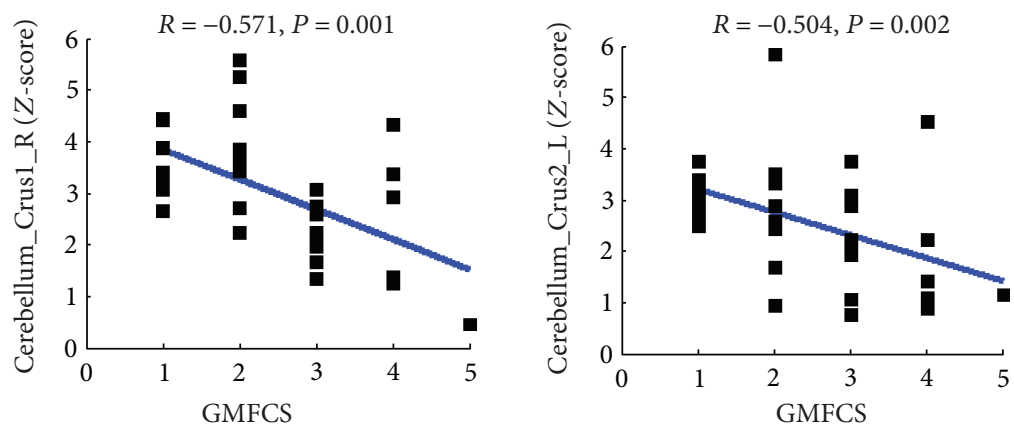

(a) Relationship between the RSN and GMFCS
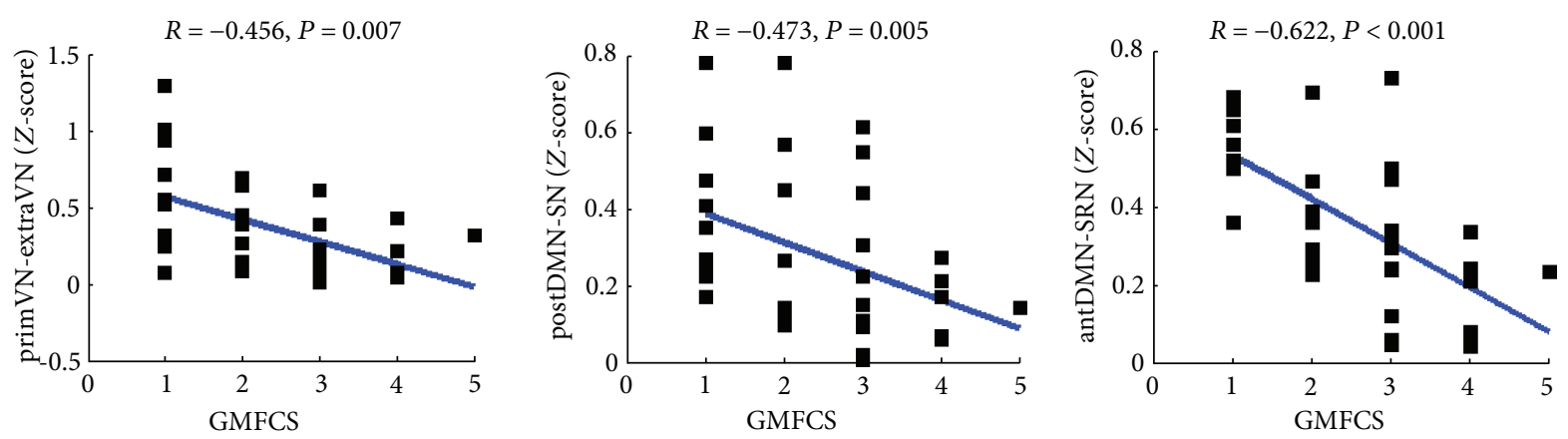

(b) Relationship between the FNC and GMFCS
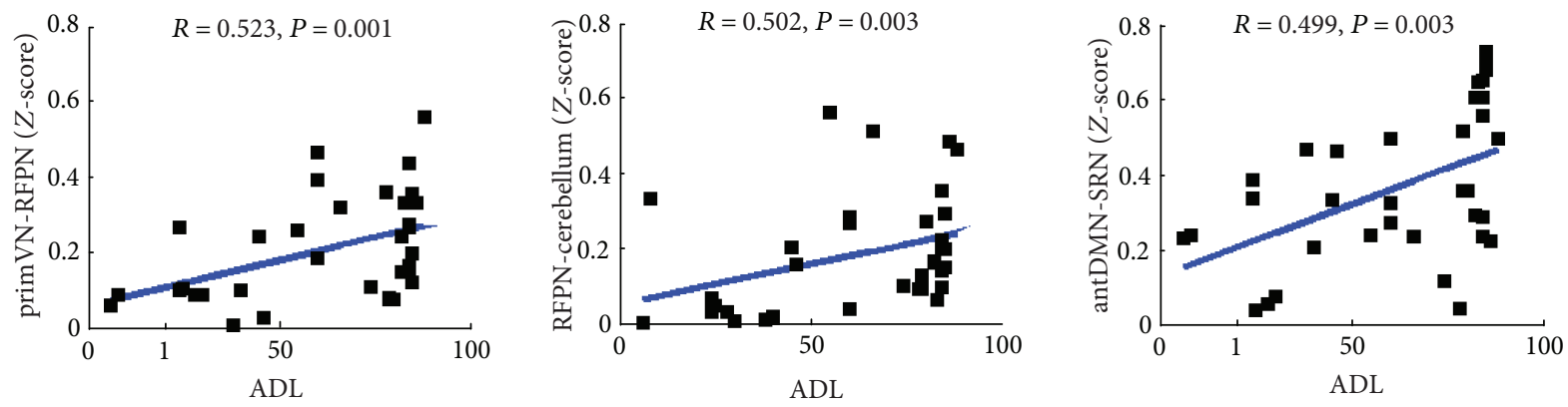

(c) Relationship between the FNC and ADL

FIGURE 4: (a) Relationship between cerebellum network and GMFCS scores. (b) Relationship between primVN-extraVN, postDMN-SN, antDMN-SRN connection, and GMFCS scores. (c) Relationship between primVN-RFPN, RFPN-cerebellum, antDMN-SRN connection, and ADL scores.

study may correlate with the impaired executive function in $\mathrm{CP}$, while the increased connectivity in the ACC may be the result of plasticity and compensation mechanism during development.

In the current study, common FNC alteration in DCP and SCP was revealed. Disrupted connectivity for primVNextraVN, as well as primVN-RFPN connection, was found both in DCP and SCP. Visual perception dysfunction reflects an impairment of the capacity to process visual information, which frequently occurs in cerebral palsy and preterm children $[10,69]$. As the frontoparietal network is an important network in visual-spatial attention [61], the disconnection of primVN-RFPN pathway might be one factor of visual perception impairment in CP. Moreover, decreased connectivity was exhibited in postDMN-SN and postDMN-LFPN in both DCP and SCP. The DMN, which has been studied extensively, has a set of brain regions that typically activate at rest but deactivate during performance of cognitive tasks $[52,70]$. $\mathrm{SN}$ and LFPN are both described as task-positive networks, with activation in corresponding areas during cognitive task performance [64]. The interaction among DMN, SN, and LFPN might be related to the low-frequency toggling between the introspective and extrospective states that ensures that the individual is attentive to the unexpected or novel environmental events [71].The decreased functional connectivity between the DMN and SN/LFPN implicated the inefficient balance and regulation among these networks, which may suggest network inhibition or network unbalance as a plausible mechanism for cognitive abnormality in children with CP.

Two distinct functional disconnections were found in the DCP group compared with the SCP group, that is, cerebellum-RFPN and antDMN-SRN connections. RFPN is a network related to visual-spatial attention and somesthetic 
perception, while the cerebellum is the center of motor planning and motor control. Their connection, as one part of motor pathway in the brain [22], may be a vulnerable link related with the dystonic DCP. As both DMN and SRN are associated with the psychological functions of introspective and self-referential processing $[52,71,72]$, the discoupling between the antDMN and SRN might relate to the disrupted cognitive self-regulation in children with DCP $[73,74]$. Moreover, negative correlation between the antDMN-SRN and GMFCS, as well as positive correlations between RFPN-cerebellum, antDMN-SRN, and ADL scores, suggested that these disconnections also contribute to motor and daily activities' dysfunction in children with CP.

In this study, some children in the $\mathrm{CP}$ group had gray matter abnormality, and children with DCP recruited in our study tended to have more severe motion and cognitive disability compared to the SCP group. In order to investigate the effect of gray matter lesions, we took the ratio of the gray matter volume of each RSN to the TIV into consideration as covariance and reconducted the post hoc $t$-test to examine the group difference between DCP and SCP in FNC analysis. The same disconnections (cerebellum-RFPN and antDMNSRN connection) were found in the DCP group compared with SCP. These results suggested that gray matter volume did not have a negative impact on the functional connectivity values in this study, mainly due to the strict inclusion criteria that the children with serious brain loss or lesions have been excluded.

Structure effect is one common concern in $\mathrm{CP}$ functional studies. Severe lesions will certainly distort the brain functional connectivity and have adverse effect in group analysis. Although children with severe brain lesions and complex clinical features were excluded in this study, potential structural effect may be inevitable. In this study, two valid measures were applied in order to weaken the effect of lesion structure to functional connectivity. On one hand, gray template mask without lesion was applied. One the other hand, ICA detects interacting networks of regions and offers the advantage to diminish the effect of abnormal signal of voxel level. During the dual-regression (temporal and spatial) back reconstruction, abnormal time courses of lesions would have slight contribution to the individual IC networks [75]. Furthermore, the FNC comparison between two $\mathrm{CP}$ groups, using the ratio of the gray matter volume of each network to the TIV as covariance, demonstrated slight influence of gray matter volume variation. The result suggested that the combination of participant exclusion criterion, the methodology, and reasonable template without lesions was capable to weaken the adverse impact of structural lesions during fMRI studies.

\section{Limitations}

However, there do exist some methodological issues and limitations that may affect our results. Firstly, there are a variety of brain lesions in the CP group. We chose the participants mainly according to the clinical diagnosis and MRI structure images, and there may be potential structural effect. In this study, some children with DCP have lesions in the putamen, and the lesions are excluded in the following analysis. However, the basal ganglia is an important motor-related network; the negligence of relative region in this study limited the functional connectivity analysis for the basal ganglia $[17,73]$. Secondly, as most of the children with $\mathrm{CP}$ are accompanied by involuntary and uncontrolled movement, it is hard for them to keep still during MRI scanning for a relatively long time. Therefore, we have to scan these children under sedation state in this study. The sedation effect on the resting-state brain is inevitable. The widely recognized effects of drug-induced sedation on RSNs were the increased connectivity within motor network and visual network and the decreased connectivity in the DMN and SN [76-78]. Although our study showing inconsistent results revealed decreased connectivity in the SMN2 and increased connectivity in the ACC of the $\mathrm{SN}$ for $\mathrm{CP}$ children under sedation condition, the influence of sedation drug on functional connectivity cannot be ignored. Also, as there were only GMFCS and ADL measurement in this study, no specificity in motion and cognitive scores was applied. In the future study, more and refined related clinical scores should be adopted. Moreover, the sample size is small in this study, and future studies should include larger sample size to determine the mechanism underlying the abnormal functional connectivity found in the current study. Finally, ICA is an unsupervised method, which remains an indirect limitation when applying to identify the RSNs. The number of independent components and the reliability of IC maps are still open questions. Although we implemented ICA at different model orders and got a relatively stable result, the individual patient-specific network combined with the seed-based functional connectivity analysis and cluster analysis might provide complementary information in the comparisons.

\section{Conclusions}

This study demonstrated the functional network alteration in children with spastic and dyskinetic cerebral palsy based on resting-state fMRI. It provided a valid tool to help elucidate the abnormal functional connectivity patterns in different $\mathrm{CP}$ subtypes. Aberrant functional connectivity in CP groups was found, particularly within the cerebellum, SMN, LFPN, and SN networks, covering the motor- and cognitiverelated networks. For FNC analysis, altered visual pathway and the covariance of DMN with other networks may be important factors of the visual perception and cognitive impairment in CP. In addition, distinct disconnections were revealed in DCP between the cerebellum and RFPN, as well as between the antDMN and SRN, which may act as an objective indicator of the clinical response in DCP. Exploring the common and distinct functional connectivity alteration is therefore beneficial for understanding the underlying mechanism of different $\mathrm{CP}$ subtypes and may contribute to more appropriate interventions.

\section{Abbreviations}

CP: $\quad$ Cerebral palsy

SCP: $\quad$ Spastic cerebral palsy 
DCP: Dyskinetic cerebral palsy

RSNs: Resting-state networks

FNC: $\quad$ Functional network connectivity

GMFCS: Gross Motor Function Classification System

ADL: $\quad$ Everyday activities of daily living

SMN1: Sensorimotor network 1, included the paracentral lobule, the supplementary motor area, and preand postcentral gyrus

SMN2: Sensorimotor network 2, focused at the lateral primary somatosensory cortex, including pre- and postcentral gyrus areas

primVN: The primary visual network

extraVN: The extrastriate visual network.

\section{Data Availability}

The data used to support the findings of this study are available from the corresponding author upon request.

\section{Ethical Approval}

Parents of all participants gave written informed consent in accordance with the Declaration of Helsinki. This study was performed according to the guidelines approved by the Ethics Committee of the University of Electronic Science and Technology of China (UESTC).

\section{Conflicts of Interest}

None of the authors has any conflict of interests to disclose.

\section{Authors' Contributions}

Yun Qin, Hui He, Cheng Lu, and Chengyan Sun conceived and designed the work. Rui Peng, Yanan Li, and Jianfu Li acquired the data. Yun Qin, Hui He, Cheng Lu, and Bo Sun analyzed the data. Yun Qin and Tao Zhang wrote the paper. All authors revised the work for important intellectual content. All of the authors have read and approved the manuscript.

\section{Acknowledgments}

This work was supported by grants from the National Nature Science Foundation of China (Grant nos. 81330032, 81771822), Special-Funded Program on National Key Scientific Instruments and Equipment Development of China (no. 2013YQ490859), the Chinese Fundamental Research Funding for the Central Universities in the University of Electronic Science and Technology of China (ZYGX2015J091, ZYGX2016J124), and the Project of Science and Technology Department of Sichuan Province (2017HH0001 and 2017SZ0004). Thanks are due to the two radiologists for the neuroimaging evaluation and to Benjamin Klugah-Brown for the English language improvement.

\section{Supplementary Materials}

The group-level spatial maps of 14 RSNs for HC, SCP, and DCP and the relationships between RSN/FNC and clinical scores in the subgroups. (Supplementary Materials)

\section{References}

[1] I. Novak, M. Hines, S. Goldsmith, and R. Barclay, "Clinical prognostic messages from a systematic review on cerebral palsy," Pediatrics, vol. 130, no. 5, pp. e1285-e1312, 2012.

[2] M. Oskoui, F. Coutinho, J. Dykeman, N. Jetté, and T. Pringsheim, "An update on the prevalence of cerebral palsy: a systematic review and meta-analysis," Developmental Medicine \& Child Neurology, vol. 55, no. 6, pp. 509-519, 2013.

[3] M. Bax, M. Goldstein, P. Rosenbaum et al., "Proposed definition and classification of cerebral palsy, April 2005," Developmental Medicine and Child Neurology, vol. 47, no. 8, pp. 571-576, 2005.

[4] P. Rosenbaum, "The definition and classification of cerebral palsy: are we any further ahead in 2006?"” NeoReviews, vol. 7, no. 11, pp. e569-e574, 2006.

[5] P. I. Tomlin, The StaticEncephalopathies, Times-Wolfe International, London, 1995.

[6] M. Bax, C. Tydeman, and O. Flodmark, "Clinical and MRI correlates of cerebral palsy," JAMA, vol. 296, no. 13, pp. 1602 1608, 2006.

[7] C. Cans, H. Dolk, M. Platt, A. Colver, A. Prasauskene, and I. K. Rägeloh-Mann, "Recommendations from the SCPE collaborative group for defining and classifying cerebral palsy," Developmental Medicine \& Child Neurology, vol. 49, pp. 35-38, 2007.

[8] T. D. Sanger, D. Chen, D. L. Fehlings et al., "Definition and classification of hyperkinetic movements in childhood," Movement Disorders, vol. 25, no. 11, pp. 1538-1549, 2010.

[9] L. Bottcher, E. M. Flachs, and P. Uldall, "Attentional and executive impairments in children with spastic cerebral palsy," Developmental Medicine \& Child Neurology, vol. 52, no. 2, article e42, e47 pages, 2010.

[10] A. Ego, K. Lidzba, P. Brovedani et al., "Visual-perceptual impairment in children with cerebral palsy: a systematic review," Developmental Medicine and Child Neurology, vol. 57, pp. 46-51, 2015.

[11] D. S. Reilly, M. H. Woollacott, P. van Donkelaar, and S. Saavedra, "The interaction between executive attention and postural control in dual-task conditions: children with cerebral palsy," Archives of Physical Medicine and Rehabilitation, vol. 89, no. 5, pp. 834-842, 2008.

[12] C. Papadelis, B. Ahtam, M. Nazarova et al., "Cortical somatosensory reorganization in children with spastic cerebral palsy: a multimodal neuroimaging study," Frontiers in Human Neuroscience, vol. 8, p. 725, 2014.

[13] K. Himmelmann and P. Uvebrant, "Function and neuroimaging in cerebral palsy: a population-based study," Developmental Medicine \& Child Neurology, vol. 53, no. 6, pp. 516-521, 2011.

[14] S. Yoshida, K. Hayakawa, K. Oishi et al., "Athetotic and spastic cerebral palsy:anatomic characterization based on diffusiontensor imaging," Radiology, vol. 260, no. 2, pp. 511-520, 2011.

[15] A. Okumura, T. Kato, K. Kuno, F. Hayakawa, and K. Watanabe, "MRI findings in patients with spastic cerebral palsy. II: correlation with type of cerebral palsy," 
Developmental Medicine \& Child Neurology, vol. 39, no. 6, pp. 369-372, 1997.

[16] M. Hadders-Algra, "Early diagnosis and early intervention in cerebral palsy," Frontiers in Neurology, vol. 5, 2014.

[17] B. R. Aravamuthan and J. L. Waugh, "Localization of basal ganglia and thalamic damage in dyskinetic cerebral palsy," Pediatric Neurology, vol. 54, pp. 11-21, 2016.

[18] I. Krägeloh-Mann and C. Cans, "Cerebral palsy update," Brain \& Development, vol. 31, no. 7, pp. 537-544, 2009.

[19] A. H. Hoon, W. T. Lawrie, E. R. Melhem et al., "Diffusion tensor imaging of periventricular leukomalacia shows affected sensory cortex white matter pathways," Neurology, vol. 59, no. 5, pp. 752-756, 2002.

[20] B. Thomas, M. Eyssen, R. Peeters et al., "Quantitative diffusion tensor imaging in cerebral palsy due to periventricular white matter injury,” Brain, vol. 128, no. 11, pp. 2562-2577, 2005.

[21] R. Trivedi, S. Agarwal, V. Shah et al., "Correlation of quantitative sensorimotor tractography with clinical grade of cerebral palsy," Neuroradiology, vol. 52, no. 8, pp. 759-765, 2010.

[22] J. D. Lee, H. J. Park, E. S. Park et al., "Motor pathway injury in patients with periventricular leucomalacia and spastic diplegia," Brain, vol. 134, no. 4, pp. 1199-1210, 2011.

[23] A. M. Pagnozzi, K. Shen, J. D. Doecke et al., "Using ventricular modeling to robustly probe significant deep gray matter pathologies: application to cerebral palsy," Human Brain Mapping, vol. 37, no. 11, pp. 3795-3809, 2016.

[24] S. M. Reid, C. D. Dagia, M. R. Ditchfield, and D. S. Reddihough, "Grey matter injury patterns in cerebral palsy: associations between structural involvement on MRI and clinical outcomes," Developmental Medicine and Child Neurology, vol. 57, no. 12, pp. 1159-1167, 2015.

[25] J. Accardo, H. Kammann, and A. H. Hoon Jr, "Neuroimaging in cerebral palsy," The Journal of Pediatrics, vol. 145, no. 2, pp. S19-S27, 2004.

[26] E. Chinier, S. N'Guyen, G. Lignon, A. ter Minassian, I. Richard, and M. Dinomais, "Effect of motor imagery in children with unilateral cerebral palsy: fMRI study," PLoS One, vol. 9, no. 4, article e93378, 2014.

[27] K. Lidzba, M. Staudt, M. Wilke, and I. Krageloh-Mann, "Visuospatial deficits in patients with early left-hemispheric lesions and functional reorganization of language: consequence of lesion or reorganization?", Neuropsychologia, vol. 44, no. 7, pp. 1088-1094, 2006.

[28] J. G. Ojemann, R. C. McKinstry, P. Mukherjee, T. S. Park, and H. Burton, "Hand somatosensory cortex activity following selective dorsal rhizotomy: report of three cases with fMRI," Child's Nervous System, vol. 21, no. 2, pp. 115-121, 2005.

[29] J. R. Wingert, R. J. Sinclair, S. Dixit, D. L. Damiano, and H. Burton, "Somatosensory-evoked cortical activity in spastic diplegic cerebral palsy," Human Brain Mapping, vol. 31, no. 11, pp. 1772-1785, 2010.

[30] L. Bottcher, "Children with spastic cerebral palsy, their cognitive functioning, and social participation: a review," Child Neuropsychology, vol. 16, no. 3, pp. 209-228, 2010.

[31] S. M. Smith, P. T. Fox, K. L. Miller et al., "Correspondence of the brain's functional architecture during activation and rest," Proceedings of the National Academy of Sciences of the United States of America, vol. 106, no. 31, pp. 1304013045, 2009.

[32] H. Burton, S. Dixit, P. Litkowski, and J. R. Wingert, "Functional connectivity for somatosensory and motor cortex in spastic diplegia," Somatosensory \& Motor Research, vol. 26, no. 4, pp. 90-104, 2010.

[33] X. Mu, Z. Wang, B. Nie et al., "Altered regional and circuit resting-state activity in patients with occult spastic diplegic cerebral palsy," Pediatrics \& Neonatology, 2017.

[34] M. de Luca, C. F. Beckmann, N. de Stefano, P. M. Matthews, and S. M. Smith, "fMRI resting state networks define distinct modes of long-distance interactions in the human brain," NeuroImage, vol. 29, no. 4, pp. 1359-1367, 2006.

[35] M. D. Fox and M. E. Raichle, "Spontaneous fluctuations in brain activity observed with functional magnetic resonance imaging," Nature Reviews Neuroscience, vol. 8, no. 9, pp. 700-711, 2007.

[36] M. J. Jafri, G. D. Pearlson, M. Stevens, and V. D. Calhoun, “A method for functional network connectivity among spatially independent resting-state components in schizophrenia," NeuroImage, vol. 39, no. 4, pp. 1666-1681, 2008.

[37] A. Abou Elseoud, H. Littow, J. Remes et al., "Group-ICA model order highlights patterns of functional brain connectivity," Frontiers in Systems Neuroscience, vol. 5, p. 37, 2011.

[38] V. D. Calhoun, T. Adali, G. D. Pearlson, and J. J. Pekar, “A method for making group inferences from functional MRI data using independent component analysis," Human Brain Mapping, vol. 14, no. 3, pp. 140-151, 2001.

[39] R. J. Palisano, P. Rosenbaum, D. Bartlett, and M. H. Livingston, "Content validity of the expanded and revised gross motor function classification system," Developmental Medicine \& Child Neurology, vol. 50, no. 10, pp. 744-750, 2008.

[40] B. R. van Zelst, M. D. Miller, R. N. Russo, S. Murchland, and M. Crotty, "Activities of daily living in children with hemiplegic cerebral palsy: a cross-sectional evaluation using the assessment of motor and process skills," Developmental Medicine \& Child Neurology, vol. 48, no. 9, pp. 723-727, 2006.

[41] L. An, Q. J. Cao, M. Q. Sui et al., "Local synchronization and amplitude of the fluctuation of spontaneous brain activity in attention-deficit/hyperactivity disorder: a resting-state fMRI study," Neuroscience Bulletin, vol. 29, no. 5, pp. 603-613, 2013.

[42] X. Bai, M. Vestal, R. Berman et al., "Dynamic time course of typical childhood absence seizures: EEG, behavior, and functional magnetic resonance imaging," Journal of Neuroscience, vol. 30, no. 17, pp. 5884-5893, 2010.

[43] M. Dinomais, G. Lignon, E. Chinier, I. Richard, A. ter Minassian, and S. N.'. G. T. Tich, "Effect of observation of simple hand movement on brain activations in patients with unilateral cerebral palsy: an fMRI study," Research in Developmental Disabilities, vol. 34, no. 6, pp. 1928-1937, 2013.

[44] J. D. Power, K. A. Barnes, A. Z. Snyder, B. L. Schlaggar, and S. E. Petersen, "Spurious but systematic correlations in functional connectivity MRI networks arise from subject motion," NeuroImage, vol. 59, no. 3, pp. 2142-2154, 2012.

[45] M. Brett, A. P. Leff, C. Rorden, and J. Ashburner, "Spatial normalization of brain images with focal lesions using cost function masking," NeuroImage, vol. 14, no. 2, pp. 486-500, 2001.

[46] M. Yang, J. Li, Y. Li et al., "Altered intrinsic regional activity and interregional functional connectivity in post-stroke aphasia," Scientific Reports, vol. 6, no. 1, article 24803, 2016.

[47] Y.-O. Li, T. Adal1, and V. D. Calhoun, "Estimating the number of independent components for functional magnetic resonance imaging data," Human Brain Mapping, vol. 28, no. 11, pp. 1251-1266, 2007. 
[48] H. Zhang, X. N. Zuo, S. Y. Ma, Y. F. Zang, M. P. Milham, and C. Z. Zhu, "Subject order-independent group ICA (SOIGICA) for functional MRI data analysis," NeuroImage, vol. 51, no. 4, pp. 1414-1424, 2010.

[49] N. U. F. Dosenbach, D. A. Fair, F. M. Miezin et al., "Distinct brain networks for adaptive and stable task control in humans," Proceedings of the National Academy of Sciences of the United States of America, vol. 104, no. 26, pp. 1107311078, 2007.

[50] M. D. Fox, A. Z. Snyder, J. L. Vincent, M. Corbetta, D. C. Van Essen, and M. E. Raichle, "The human brain is intrinsically organized into dynamic, anticorrelated functional networks," Proceedings of the National Academy of Sciences of the United States of America, vol. 102, no. 27, pp. 9673-9678, 2005.

[51] C. Luo, Q. Li, Y. Lai et al., "Altered functional connectivity in default mode network in absence epilepsy: a resting-state fMRI study," Human Brain Mapping, vol. 32, no. 3, pp. 438-449, 2011.

[52] C. Luo, C. Qiu, Z. Guo et al., "Disrupted functional brain connectivity in partial epilepsy: a resting-state fMRI study," PLoS One, vol. 7, no. 1, article e28196, 2012.

[53] C. F. Beckmann, M. Deluca, J. T. Devlin, and S. M. Smith, "Investigations into resting-state connectivity using independent component analysis," Philosophical Transactions of the Royal Society B: Biological Sciences, vol. 360, no. 1457, pp. 1001-1013, 2005.

[54] M. Allin, H. Matsumoto, A. M. Santhouse et al., "Cognitive and motor function and the size of the cerebellum in adolescents born very pre-term," Brain, vol. 124, no. 1, pp. 60-66, 2001.

[55] M. Rapoport, R. van Reekum, and H. Mayberg, "The role of the cerebellum in cognition and behavior: a selective review," Journal of Neuropsychiatry \& Clinical Neurosciences, vol. 12, no. 2, pp. 193-198, 2000.

[56] W. T. Thach, "What is the role of the cerebellum in motor learning and cognition?," Trends in Cognitive Sciences, vol. 2, no. 9, pp. 331-337, 1998.

[57] W. Kułak and W. Sobaniec, "Magnetic resonance imaging of the cerebellum and brain stem in children with cerebral palsy," Advances in Medical Sciences, vol. 52, Supplement 1, pp. 180182, 2007.

[58] R. Davis, "Cerebellar stimulation for cerebral palsy spasticity, function, and seizures," Archives of Medical Research, vol. 31, no. 3, pp. 290-299, 2000.

[59] G. Abbruzzese and A. Berardelli, "Further progress in understanding the pathophysiology of primary dystonia," Movement Disorders, vol. 26, no. 7, pp. 1185-1186, 2011.

[60] H. Tsao, K. Pannek, R. N. Boyd, and S. E. Rose, "Changes in the integrity of thalamocortical connections are associated with sensorimotor deficits in children with congenital hemiplegia," Brain Structure and Function, vol. 220, no. 1, pp. 307-318, 2015.

[61] M. Corbetta, "Frontoparietal cortical networks for directing attention and the eye to visual locations: identical, independent, or overlapping neural systems?," Proceedings of the National Academy of Sciences of the United States of America, vol. 95, no. 3, pp. 831-838, 1998.

[62] H. Bassan, C. B. Benson, C. Limperopoulos et al., "Ultrasonographic features and severity scoring of periventricular hemorrhagic infarction in relation to risk factors and outcome," Pediatrics, vol. 117, no. 6, pp. 2111-2118, 2006.
[63] E. Roze, "Risk factors for adverse outcome in preterm infants with periventricular hemorrhagic infarction," Pediatrics, vol. 122, no. 1, pp. e46-e52, 2008.

[64] V. Bonnelle, T. E. Ham, R. Leech et al., "Salience network integrity predicts default mode network function after traumatic brain injury," Proceedings of the National Academy of Sciences of the United States of America, vol. 109, no. 12, pp. 4690-4695, 2012.

[65] W. W. Seeley, V. Menon, A. F. Schatzberg et al., "Dissociable intrinsic connectivity networks for salience processing and executive control," The Journal of Neuroscience, vol. 27, no. 9, pp. 2349-2356, 2007.

[66] F. A. Mansouri, T. Egner, and M. J. Buckley, "Monitoring demands for executive control: shared functions between human and nonhuman primates," Trends in Neurosciences, vol. 40, no. 1, pp. 15-27, 2017.

[67] E. K. Miller and J. D. Cohen, "An integrative theory of prefrontal cortex function," Annual Review of Neuroscience, vol. 24, no. 1, pp. 167-202, 2001.

[68] C. S. Carter, A. M. Macdonald, M. Botvinick et al., "Parsing executive processes: strategic vs. evaluative functions of the anterior cingulate cortex," Proceedings of the National Academy of Sciences of the United States of America, vol. 97, no. 4, pp. 1944-1948, 2000.

[69] E. Fazzi, S. Bova, A. Giovenzana, S. Signorini, C. Uggetti, and P. Bianchi, "Cognitive visual dysfunctions in preterm children with periventricular leukomalacia," Developmental Medicine and Child Neurology, vol. 51, no. 12, pp. 974-981, 2009.

[70] M. E. Raichle, A. M. MacLeod, A. Z. Snyder, W. J. Powers, D. A. Gusnard, and G. L. Shulman, "A default mode of brain function," Proceedings of the National Academy of Sciences of the United States of America, vol. 98, no. 2, pp. 676-682, 2001.

[71] S. J. Broyd, C. Demanuele, S. Debener, S. K. Helps, C. J. James, and E. J. S. Sonuga-Barke, "Default-mode brain dysfunction in mental disorders: a systematic review," Neuroscience and Biobehavioral Reviews, vol. 33, no. 3, pp. 279-296, 2009.

[72] A. D'Argembeau, F. Collette, M. van der Linden et al., "Selfreferential reflective activity and its relationship with rest: a PET study," NeuroImage, vol. 25, no. 2, pp. 616-624, 2005.

[73] K. Himmelmann, "The quest for patterns in dyskinetic cerebral palsy," Developmental Medicine \& Child Neurology, vol. 58, no. 2, pp. 112-112, 2016.

[74] K. Himmelmann, G. Hagberg, L. M. Wiklund, M. N. Eek, and P. Uvebrant, "Dyskinetic cerebral palsy: a population-based study of children born between 1991 and 1998," Developmental Medicine \& Child Neurology, vol. 49, no. 4, pp. 246-251, 2007.

[75] X. N. Zuo, C. Kelly, J. S. Adelstein, D. F. Klein, F. X. Castellanos, and M. P. Milham, "Reliable intrinsic connectivity networks: test-retest evaluation using ICA and dual regression approach," NeuroImage, vol. 49, no. 3, pp. 2163-2177, 2010.

[76] A. G. Hudetz, "General anesthesia and human brain connectivity,” Brain Connectivity, vol. 2, no. 6, pp. 291-302, 2012.

[77] P. Liang, H. Zhang, Y. Xu, W. Jia, Y. Zang, and K. Li, “Disruption of cortical integration during midazolam-induced light sedation," Human Brain Mapping, vol. 36, no. 11, pp. 42474261, 2015.

[78] M. Qiu, D. Scheinost, R. Ramani, and R. T. Constable, "Multi-modal analysis of functional connectivity and cerebral blood flow reveals shared and unique effects of propofol in large-scale brain networks," NeuroImage, vol. 148, pp. 130-140, 2017. 


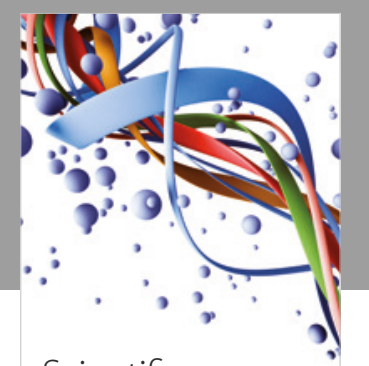

Scientifica
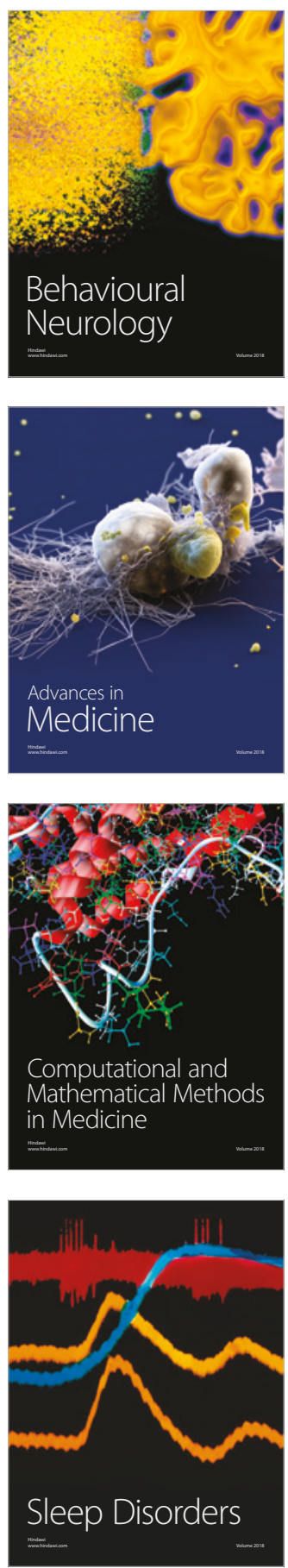

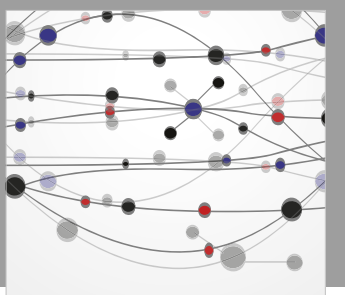

The Scientific World Journal

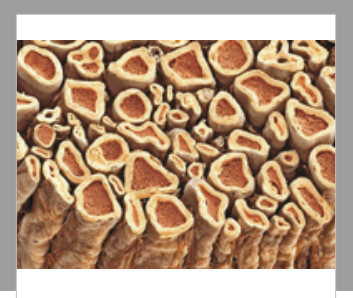

Case Reports in

Neurological Medicine

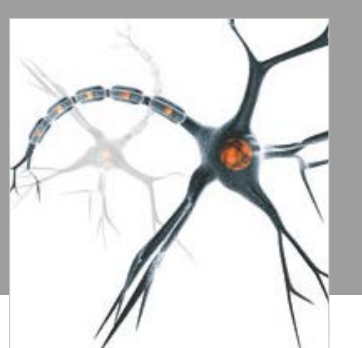

Neural Plasticity

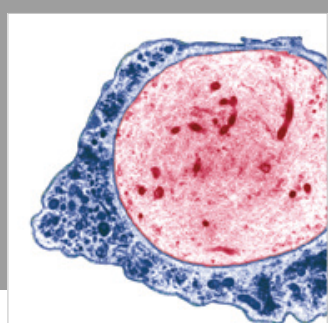

Multiple Sclerosis

International

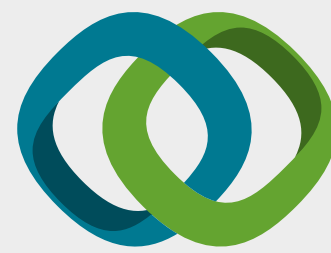

Hindawi

Submit your manuscripts at

www.hindawi.com
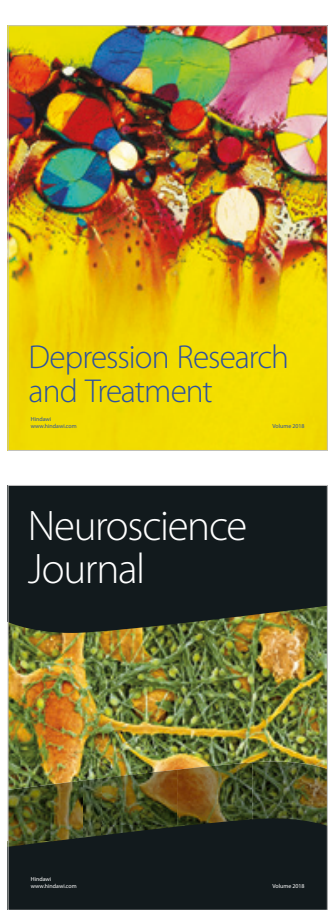

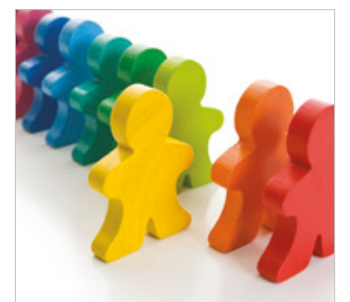

Autism

Research and Treatment
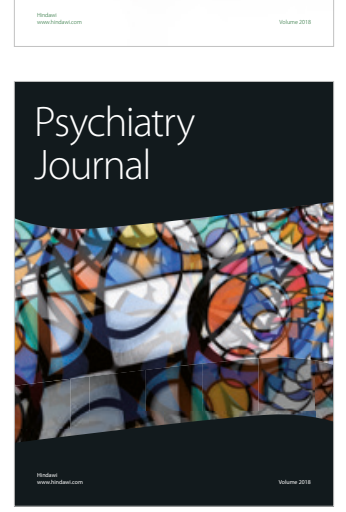
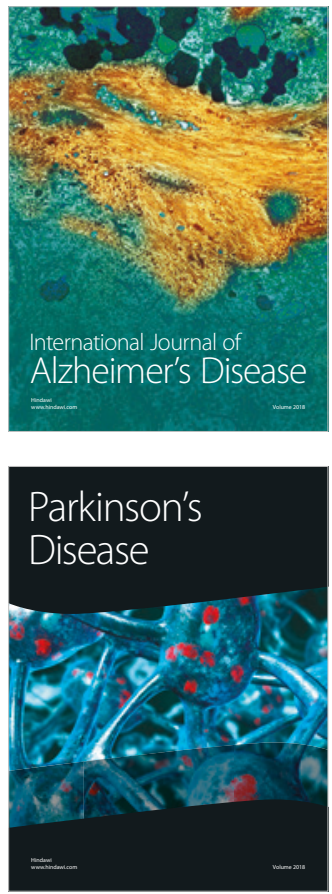
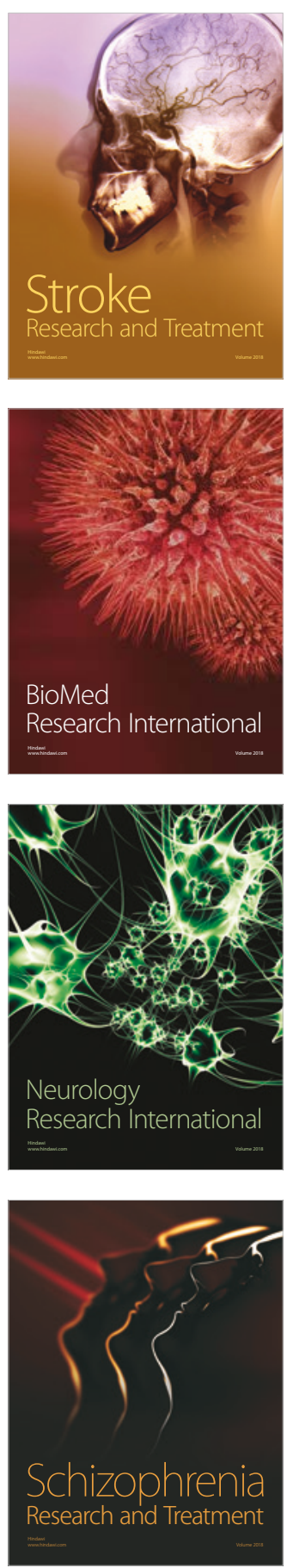